\title{
Indexically Structured Ecological Communities
}

\begin{abstract}
Ecological communities are seldom, if ever, biological individuals. They lack causal boundaries as the populations that constitute communities are not congruent and rarely have persistent functional roles regulating the communities' higher properties. Instead we should represent ecological communities indexically, by identifying ecological communities via the network of weak causal interactions between populations that unfurl from a starting set of populations. This precisification of ecological communities helps identify how community properties remain invariant, and why they have robust characteristics. This is a more general framework than individuality, respecting the diversity and aggregational nature of these complex systems while still vindicating them as units worthy of investigation.
\end{abstract}

Word Count: 4955 


\section{Introduction}

Ecology studies the distribution and abundance of populations across landscapes and over time. Community ecology has long operated with the assumption of "local determinism": i.e. that ecological patterns are primarily explained by the interaction of local populations within a community. The 'local ecological community' functions as the core unit of investigation; it is thought to have discrete boundaries, stable composition, predictable dynamics over time, and allows for inferences made from one community to apply to the next. But there have been many dissenting voices within the ecological research tradition who instead argue for ecological individualism, emphasizing that populations generally move around a landscape of their own accord driven by chance and by abiotic factors and are not heavily influenced by their local neighbours and as a result ecological communities are largely ephemeral compositions of populations. This debate drives considerations whether there are law-like regularities in community ecology.

To arbitrate this debate philosophers and biologists have provided an analyses of the conditions for an assemblage- a collection of populations in a space- to be an ecological community. Namely, assemblages should be a biological individual just like an individual organism or a lineage. If an ecological community is a biological individual then it is the cohesive and distinct entity that local determinism presupposes. Jay Odenbaugh and Kim Sterelny independently specified the conditions under which an ecological assemblage can be thought of as an objective and important unit in nature, an ecological community. Both authors leave it empirically open as to whether and which assemblages satisfy the conditions they present (Odenbaugh, 2007; Sterelny; 2006).

I argue that as ecological communities so rarely satisfy these conditions we need an alternative account of ecological communities. Instead ecological systems are largely 
aggregations of individual populations unlinked by stable, strong causal interactions. As a result they are better described indexically, as causal networks which unfurl from a specific point of reference. This acts to fix the reference in these unsystematic systems and allows for the identification of the robust parts and robust properties of ecological systems. To infer from one community to the next we need a precise account of the identity of the units we are discussing. This elaboration on indexical communities provides that. This is not to say that ecological communities will never be biological individual, there will be limiting cases. But these lie so far from the norm that we need a framework that better represent the degree of variation in ecological assemblages.

This proposal provides a substantively different framework to biological individuality, diversifying the ontological toolkit of philosophers of biology. To do this I will first introduce the theory behind ecological communities as biological individuals. I then elaborate on what it takes for a collection of parts to be an individual and why ecological systems are almost always not. Into this lacuna I then present the indexical account of ecological communities and the advantages it entails.

\section{Communities as Biological Individuals}

Multicellular biological individuals evolved from single cell biological individuals. While multicellular individuals often evolve from a single species population it is not uncommon for multispecies assemblages to form individual organisms such as lichens ${ }^{1}$. But there is also integration without unification; most large metazoans only function by inheriting bacteria that maintain them in a symbiotic relationship (perhaps also some biofilms). These biological

\footnotetext{
${ }^{1}$ Single species populations transitioning into an individual are referred to as fraternal transitions. Multispecies transitions are referred to as egalitarian transitions (Queller, 2000).
} 
phenomena demonstrate that strong ecological interactions precede transitions in individuality and multispecies assemblages can be individuals. But where does this leave the assemblage's community ecologists are familiar with? They are clearly less of an individual than populations in close symbiotic relationships. Any account of biological individuality for communities needs to be able to account for degrees of individuality. One way to indicate this is by providing a set of conditions which if fulfilled rightly counts an ecological community as an individual, then leave it open as to whether any actual ecological community satisfies these conditions. This is what both Sterelny and Odenbaugh do. The conditions they present follow:

\subsection{Boundaries}

Individuals, as spatio-temporal entities comprised of interacting sub-parts, have boundaries. For interacting parts to be a whole there must be strong causal interactions creating internal cohesion within the system which isolates it from external influences. The system parts in community ecology are the populations which causally interact, creating feedback loops maintaining local populations and excluding external populations from invading the local system. Sterelny particularly notes that local niche construction is one way populations can maintain an assemblage. Famously, Australian plants including Gums, Banksias, and Melaleucas are adapted to fire and facilitate the presence of each other by making their local environment more fire prone. Under this conception of boundaries ecological communities are bound by interaction strength between populations (Levins and Lewontin, 1985). While this does not necessarily mean that populations in the system will be congruent, strong causal interaction is associated with spatial overlap so congruence of community populations is expected. 


\subsection{Internally Structured}

The populations that belong to an ecological individual should act in ways that police the identity of that individual. Interspecific interactions- such as predation, competition, and, mutualism- are thought to form a lattice of positive and negative feedback loops, regulating the community and creating stability. When you couple these interactions with stable geographic ranges of the populations you gain a picture of stable economy of nature in which there is persistence of local population identity due to the specific roles that these populations play. Internal structure is the product of both feedback loops that act to maintain population identity in an area and the persistence of specific populations playing particular roles in this local community.

\subsection{System-level Properties}

If we wish to include local ecological communities in our general scientific ontology there has to be a reason to talk about communities rather than just talking about the populations that make up communities. There should be predicates and properties which are needed for describing phenomena at the community level. System-level properties are an explanadum to be explained by the assemblage and an explanans for ecological and evolutionary hypotheses. Properties generally discussed on the community level are associated with the maintenance of multi-species interaction networks (community network structures), the maintenance of composition identity or aggregative features (emergent community properties), or the various material outputs that the joint assemblage create (community outputs). Odenbaugh treats system-level properties as necessary for community existence: 'species populations form an ecological community just in case... they possess a community level property' (pg 636). He primarily mentions interspecific interactions and the feedback loops they create as community level properties. Sterelny describes emergent community properties, identifying several candidate emergent properties from the diversity-stability hypothesis such as 
community population stability and community biomass production. The productivity and abiotic features ecological communities produce have become an area of keen interest for conservation science. Many ecologists have attempted to justify the preservation of ecological communities by appealing to the 'ecosystem services' - capacities commonly attributed to the community as a whole - which they provide. These system-level properties feature in ecological explanation and therefore need to be able to be represented by an account of ecological communities.

\section{Problems with Individuality}

Sterelny- Odenbaugh individuality features a tripartite criteria that ecological communities need to fulfill: they should be bound causally, they should have internal regulation, and they should have system-level properties. Sterelny represents these criteria hyper-dimensionally noting that each can be more or less instantiated (see fig 1). This is partially true, but these axes are not independent, as both authors independently note (Maclaurin and Sterelny, 2008; Odenbaugh, Forthcoming). Internal regulation demands boundaries contain regulatory patterns of interactions and system-level properties require the populations to be structured. This view implies that if communities do not have boundaries they will not have internal regulation and without internal regulation they will lack system-level properties. While I argue that communities do not have robust boundaries and their internal structure is not as stable as individuality requires, I maintain that ecological systems can have systemproperties. Loose aggregative ecological systems produce system-level properties by what I call machine robustness and ensemble robustness. 


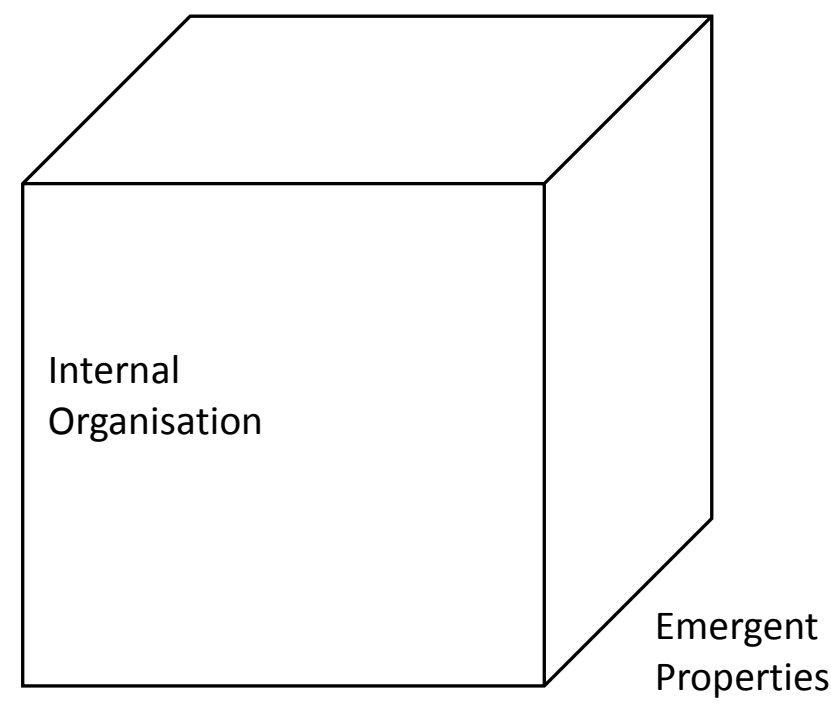

Boundaries

Figure 1. Sterelny's Multidimensional Representation of an Ecological Individual.

The best way to identify both the relation between robust outputs and the stability of the system that produces these outcomes can be found in Bill Wimsatts conceptual framework of multiple decomposition. For community boundaries to be 'real' they should be descriptively robust. An entity is descriptively robust when multiple interventions, multiple types of intervention, and different descriptions of relevant properties pick out organisation that is largely congruent (Wimsatt; 2007). The parts described by a 'decomposition' from one theoretical perspective largely align with those describe from another. By applying this procedure to local ecological communities, as I will now show, we find that communities as we often have understood them are not descriptively robust as a whole and therefore are not biological individuals.

We multiply decompose a 'local ecological community' by identifying the causal system that the different co-located populations belong to. Each population will belong to an 
ecological system consisting of just those populations to which they are counterfactually sensitive. For example identifying the ecological system that an echidna populations belongs to will include its predators, Goannas, and prey, Termites. If we claim that a local assemblage of populations belong to the same individual then those populations should map into a single ecological system. If this system is bound and has its own properties then it is a biological individual and an ecological community. If populations have a causal interaction profile which picks out the same ecological community with congruent boundaries and the same sub-parts, then that individual is robust.

The problem is that co-located populations often belong to radically different ecological systems. This is because causal relations in ecology are often asymmetrical and maps of organism distributions given by Global Information Systems (GIS) show that populations rarely spatially coincide ${ }^{2}$. Consider the factors relevant to a population of Spotted Quolls compared to their occasional prey, Greater Gliders. Individual Quolls roam over home ranges up to 3500 hectares moving between habitat fragments via wildlife corridors, while a Glider's home range is only 2 hectares and is locked within a local habitat fragment. Unless there is a very strong counterfactual dependence between these two populations the network of populations relevant to the Quolls will be radically different to the Gliders, as Quolls interact with populations that intersect with their large home ranges. Further, due to the radically different ranges and population densities there is a strong asymmetry between these populations. Differential changes in a local Glider population are unlikely to affect the Quoll population. Its range would include several Glider populations and they are generalist predators. But differential changes that increase the Quoll population would impact the

\footnotetext{
${ }^{2}$ See for example the Atlas of Living Australia for spatial distributions of populations across the continent.
} 
Glider population as increased predation can have large impacts on small local populations. This creates an asymmetry; intervention on Gliders has little impact on Quolls but intervention on Quolls significantly impacts Gliders.

Population boundaries radically differ and the causal relations between populations are often asymmetrical. When these conditions are met, congruent boundaries are rare, and identifying the population network, and the space that network occupies, will be highly dependent on the initial choice of referent. Varying the starting population or property referred to in an assemblage will yield radically different descriptions of the ecological community. Figure 2 shows the variation that can be displayed in a simple four population system for the parts and system outputs.
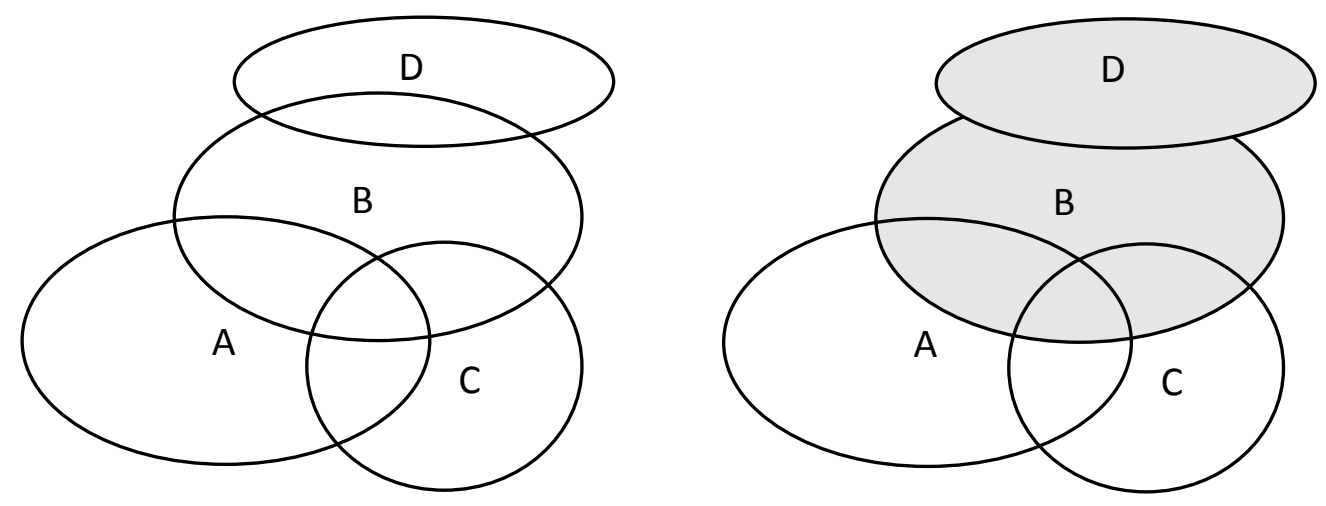

Figure 2. Asymmetry and Congruence. Each circle represents a population and its spatial range. These populations have the causal structure $\mathrm{D} \rightarrow \mathrm{B} \rightarrow \mathrm{A}, \mathrm{C} \rightarrow \mathrm{A}$. The relevant causal community of population A is all the local populations as it is causally influenced by all the other populations. If we want to find the relevant community for population B then it will include the shaded area as population B is only influenced by population D. 
Ecology aims to explain how populations and their interactions result in system level properties like diversity, stability, or ecological services, e.g. water retention and biomass production. Local determinism supposes that stable relationships between persistent populations produce these properties; stable internal structure produces system level properties. Explanations of this type are machine robust; the system-level property is a result of a particular causal sequence of interactions between persistent parts. This is, however, a problematic assumption as populations are often highly transient. In one study of 100 biomes across earth, $75 \%$ of these systems had at least one in ten species disappear locally per decade (Dornelas, Gotelli et al. 2014). This is often coupled with little change in regional diversity as populations simply shift their distribution across the larger landscape (Thuiller, Slingsby et al. 2007). These studies add further evidence to those who believe that local ecological communities are often the wrong scale to look for law-like generalities in ecology (Ricklef, 1999; Lean and Sterelny, forthcoming). They claim that regional patterns better explain the local distribution and abundance of organisms than local patterns which are ephemeral and stochastic. These views explicitly reject the idea that local community identity is primarily maintained by internal composition.

Machine robust systems are relatively rare in ecology but there are ubiquitous higher level properties begging for explanation. One way we get these stable higher level outputs is via another type of robustness commonly found in complex systems, ensemble robustness. Ensemble robustness is when the system-level property is a product of diverse and varied parts filling the same functional role. The parts in the system do not have to be identical over time and space for the high level properties to be robust. For critical feedback loops for overall system functioning we often find huge redundancy; for example gum forest pollination is done by a range of evolutionary distinct actors including marsupial, insects, and birds. The statistical aggregation of the actions of local populations can stabilize ecological 
output as a result of statistical averaging effects, biological insurance, and sampling effects (Bryant, 2010). Ecological systems also have outputs which are not just the simple aggregation of component population's actions. Diverse local species assemblages can have non-linear ecosystem outputs; for example, combinations of populations non-additively result in explosive combustion in forest fires (Van Altena et al, 2012). Due to all these factors higher level properties are ubiquitous in ecological systems even if there are no clear boundaries for these systems and the internal composition is unstable.

Despite the highly aggregational quality of ecological systems, ecological community properties are not uniformly a product of ensemble robustness; specific populations are sometimes necessary for ecological output. Keystone species, which have disproportionate impacts on assemblage composition, function like mechanisms with particular populations playing a necessary and causally specific role in maintaining whole system features. The importance of keystone species is controversial, with some ecologists pressing that there are not such strong relationships between single populations and assemblage features (Mills et al, 1993). But there is strong evidence that in some systems particular populations do play strong roles in regulating a cluster of populations in their assemblage (Ripple et al, 2001). Species can co-vary in tight relationships over geological periods that far outstretch local communities both spatially and temporally according to paleo-ecological evidence (Sterelny, 2001). Symbiotic relationships show similar tight co-variation between populations. This indicates that nested within larger assemblages we can find sets of populations with strong and persisting causal relations that are much more stable than the community as a whole.

To summarize, ecological communities are highly unsystematic systems, they lack clear boundaries and persistent internal identity, but they do have robust parts and robust system outputs via the variant aggregative interactions of their constituents. Any account of ecological community identity needs to be able to identify these explanatorily important 
properties and fix the reference of the system that produces these properties. This is difficult as population networks will not in general be congruent over different choices of starting population as small changes in referent choice can result in a quite different network. But ecological communities are still causal systems. Indexical communities describe communities via the network of causal interactions between populations and provide a way to represent their causal structure.

\section{Indexical Communities}

On a first pass of the philosophy of ecology literature, accounts of ecological communities appear to split between treating populations as largely independent of each other, or describing them within an individuality framework. There are, however, other options which sit between these extremes with Sterelny proposing 'indexical communities' in contrast to ecological individuality (Sterelny, 2006). The following account of ecological communities supplements and develops indexical communities by providing the conceptual apparatus to identify robustness and utilizing the Woodwardian interventionist framework to fix the reference of the causal system involved (Woodward, 2005).

Simple indexical communities are ecological units which aim to describe the conditions that affect the demographics of single populations. Indexically described communities are one of the most useful and utilized ecological technique in conservation science. To preserve the critically endangered Hairy Nosed Wombat we need to know how much native grasses and tubers they eat, what is an unusual parasite load, how to separate them from wild dog populations, and competing gazers. These populations are indexed to the Wombat population as they have a causal impact on them. This framework has become commonplace due in part to conservation funding being directed to individual species preservation. The science then aims to find the conditions that lead to the preservation of a focal population. 
These simple indexical communities are not thought to be very informative for community level properties as they are constructed with limited epistemic aims, i.e. explaining the influences on a single population. Due to the limited scope of such causal units they remain silent on certain, hopefully generalizable, community level features such as the relationship between diversity and stability (Sterelny, 2006). Further it is thought that information about one indexical community is difficult to apply to other assemblages due to the apparent limited nature of their scope. But we can rectify these problems by building into indexical communities the means for identifying machine robustness and ensemble robustness.

This is done by allowing for multiple decomposition of a local assemblage using Woodwardian Intervention. To be able to identify robustness in these ecological assemblage in their output or causal boundaries we need to have multiple starting points to investigate the unit. While indexical communities as they have previously been discussed are only around a focal population, this account of indexical communities expands the focal unit to a set of multiple populations. The stepwise procedure for identifying the relevant ecological community appears in Box 1, but here also is a description of the process. Take the starting set of populations and identify the indexical community of each individual population in the set. The indexical community for a population is identified by intervention: treat the focal indexical population as variable A. An alternative population variable, B, is said to be part of the same community, as well as a cause of A, if systematic intervention on B brings about change in A. Further, once we identify that a population variable has causal influence on the focal population we can ask whether intervention on populations that affect it also have 'downstream' effects on A. If so, then that population is also part of the community. Each population node introduced between the focal population and a population of interest will necessarily reduce the counterfactual relationship between them. 
Box.1. Indexical Communities can be built up from multiple indexical populations by the following procedure.

i. Define the starting set of populations and/ or a community-level property (e.g. ecosystem output).

a. If community-level property then identify the set of populations that contribute to the property.

ii. Identify the populations that are causally salient for the set of populations via intervention.

iii. Overlay the different networks of counterfactual dependencies from the specific populations.

iv. If multiple interventions pick out the same connection these are the robust relationships in a community.

This process yields a directed graphical map of the causal network indexed to population A. We repeat this procedure for all the populations in the starting set. The different causal maps are then compared. All the populations that causally contribute to the starting population are counted as part of the community. But the scope of the boundaries can be tweaked by varying the strength of the causal effect required for inclusion (Levins and Lewontin, 1985). By setting this parameter moderately high we avoid ecological holism, where each indexical community has a numerous nodes and as result each indexical community will overlap each other. Population network structures that appear from multiple different indexed populations are more robust. For example if there are populations that act like keystone species they will be part of all the directed graphs as they play necessary role in maintaining the population network structure.

What determines the starting set of populations? This is in part research interest defined but there are some obvious candidates. The first is including all the populations that cohabit 
in a location. By identifying the network of populations that emanate out of co-habiting populations we can see to what extent this local ecological community is a causally cohesive unit. Alternatively we can look at community-level properties or outputs by starting with the set of populations that are thought to contribute to this community-level feature, for instance water filtration around a lake. The ecological structure that yields this output (filtration) is of economic interest and indexical communities identify the populations that need to be preserved to maintain this community output.

What I find the most exciting aspect of this framework is it gives us the means to preserve two different important conservation units that have previously been criminally underdescribed and referentially underdetermined. These are phenomenological communities and biodiverse communities. Phenomological communities are the communities that the folk who are interested in and spend time in the environment perceive. Environmentalists and the public often have an interest in preserving particular assemblages that are familiar from their experience of the wild. These assemblages include charismatic mammals, audible bird-life, visually stimulating angiosperms, and imposing trees. To fix the reference of such local assemblages we include in the starting set the phenomenologically prominent populations in a local area. For example if you want to find the community of a Blue Gum forest you include Blue Gums, lyrebirds, and Waratahs and identify the populations relevant to them.

The second conservation-based community is a biodiverse community. The preservation of biodiversity has been the primary goal of conservation science for the last 30 years but 'biodiversity' is ill-defined. Two major philosophical positions regarding biodiversity are conventionalism - biodiversity is the features of biological difference that community stakeholders value - or realism - there are privileged carvings of biological difference which we should value (Sarkar, 2006; Maclaurin and Sterelny, 2008; Lean and Maclaurin, 2016). For 
either position we can identify the populations which represent biodiversity in that particular local area and then use this procedure to find the relevant larger ecological community.

By allowing the starting set to be determined by the interested parties we are able to tailor the indexical community to fulfil both the epistemic and normative roles that community ecology and conservation science requires.

\section{Upshots of Indexical Communities}

Built into this methodology is the means of assessing an ecological community in several ways. The first is the invariance and production of community level properties. To explain how the starting assemblage produces a particular community-level property, be it stability of population network structure or an ecosystem output like fire likelihood, we need to identify the counterfactual interventions that affect that property. We do this so we can assess the invariance of the populations and their relationship to these properties. If particular populations appear in multiple networks in the same sequence, those parts of the system are robust, so we can gain a picture of the way these stable causal relations yield community properties. The indexical community identifies the descriptively robust features of the system under inquiry. Machine robust parts of the ecological network will always be descriptively robust. Weak aggregational interactions also bring about community-level phenomena. These are instantiated by many pathways, which have modest strength. To gain a sense of the relationship between the aggregative system and these properties we need to fix the identity of the system in question. Indexical communities provides a precise way to refer to such weak 'systems' and in by doing so provides a guide for further research into the relations between populations and community-level properties.

For the ontological question of whether communities are real, indexical communities provides an answer. If the same causal structure appears from multiple starts and has robust 
boundaries then we have a robust ecological community. It is, however, more likely that we will find that we have only partial overlap between the causal maps. This acts to identify the descriptive robust sub-systems within the community. As a result, this framework provides a more fine-grained and specific way of identifying whether a particular local ecological community is a system that acts like an individual, like an organism, or an aggregate, like gas particles in a beaker. If there is no causal connections between the starting populations then this is not a unitary community. So this methodology acts not just as a descriptive tool but also an existence test. Depending on referent choice, there can be multiple precisifications of a unitary community or none.

By describing communities using a causal graph network description, we open them up to a range of formal methods of assessment. Modularity of the system and the sub-systems is one important feature. Modular clusters of causal interactors make a system more bounded and can account for particular system outputs. Formal methods like the Girvan-Newman algorithm can quantify such structures identifying modular grouping and boundaries in complex systems (Givarn and Newman, 2002).

This is all to say we can assess an indexical ecological community in terms of the invariance of its system properties, its modularity, and its descriptive robustness. If an indexical community is completely modular, descriptively robust, and has highly invariant system properties then it will be a biological individual. But communities so rarely satisfy these conditions that we need an alternative framework. There is more to biology than just the study of individuals and this proposal gives an alternative framework to describe complex biological systems. 


\section{References}

Bryant, Rachael. 2012. "What If Ecological Communities Are Not Wholes?" The Environment: Philosophy, Science, and Ethics, ed. William Kabasenche, Michael Oourke, and Matthew Slater, MIT Press: 37-56.

Dornelas, Maria., Nicholas Gotelli, Brian McGill, Hideyasi Shimadzu, Faye Moyes, Caye Sievers, and Anne Magurran 2014. "Assemblage time series reveal biodiversity change but not systematic loss." Science 344 (6181): 296-299.

Girvan, Michelle., and Mark Newman. 2002. "Community structure in social and biological networks." Proceedings of the national academy of sciences, 99(12): 7821-7826.

Lean, Christopher., and James Maclaurin. 2016. “The Value of Phylogenetic Diversity” In Biodiversity Conservation and Phylogenetic Systematics. ed. Roseli Pellens and Philippe Grandcolas, Springer Press.

Lean, Christopher., and Kim Sterelny. Forthcoming. "Biodiversity and Ecological Hierarchy." In The Routledge Handbook of the Philosophy of Biodiversity. ed. Justin Garson, Anya Plutynski, and Sahotra Sarkar

Levins, Richard., and Richard Lewontin. 1985. The Dialectical Biologist. Cambridge: Harvard University Press.

Maclaurin, James., and Kim Sterelny. 2008. What is Biodiversity? Chicago: University of Chicago Press.

Mills, Scott, Michale Soulé, and Daniel Doak. 1993. “The keystone-species concept in ecology and conservation.” BioScience, 43(4): 219-224.

Odenbaugh, Jay. 2007. "Seeing the Forest and the Trees: Realism about Communities and Ecosystems." Philosophy of Science, 74(5): 628-641. 
Odenbaugh, Jay. Forthcoming. "Conservation Biology.” The Stanford Encyclopaedia of Philosophy Ed: E. Zalta

Queller, David. 2000. "Relatedness and the fraternal major transitions.” Philosophical Transactions of the Royal Society of London B: Biological Sciences, 355(1403): 1647-1655.

Ricklefs, Robert. 2008. "Disintergration of the Ecological Community." American Naturalist 272(6): 741-750.

Ripple, William., Eric Larsen, Roy Renkin, and Douglas Smith. 2001. "Trophic cascades among wolves, elk and aspen on Yellowstone National Park's northern range." Biological Conservation, 102(3): 227-234.

Sarkar, Sahotra. 2005. Biodiversity and Environmental Philosophy. Cambridge: Cambridge University Press.

Sterelny, Kim. 2001. The reality of ecological assemblages: A palaeo-ecological puzzle. Biology and Philosophy, 16(4): 437-461.

Sterelny, Kim. 2006. "Local Ecological Communities." Philosophy of Science 73(2): 215231.

Thuiller, Wilfried., Jasper Slingsby, Sean Privett, and Richard Cowling. (2007). "Stochastic species turnover and stable coexistence in a species-rich, fire-prone plant community." Public Library Of Science One 2(9): 938.

Van Altena, Cassandra., Richard van Logtestijn, William Cornwell, and Johannes Cornelissen. 2012. Species Composition and Fire: Non-Additive Mixture Effects on Ground Fuel Flammability. Frontiers in plant science. 3:63. 
Wimsatt, William. 2007. "Complexity and Organisation." Re-Engineering Philosophy for Limited Beings: Piecewise Approximations to Reality. Cambridge: Harvard University Press, 179-192.

Woodward, James. 2005. Making things happen: A theory of causal explanation. Oxford:

Oxford University Press. 